\title{
A prediction model for pedestrian fatalities based on explanatory factors
}

\author{
Md. Minhajul Islam Khan ${ }^{1}$, Md. Hasibur Rahman ${ }^{2}$ \\ ${ }^{1,2}$ Lecturer, Department of Civil Engineering, AUST, Dhaka, Bangladesh,
}

\begin{abstract}
A study of injury severity in pedestrian accidents can help to identify the number of fatalities as a result of motor vehicle-pedestrian collisions associated with different factors. The objective of this research is to develop statistical predictive model for pedestrian fatalities at the Dhaka metropolitan, capital of Bangladesh. This research focuses on the severity of accidents i.e. number of fatalities rather than accidents number, because number of fatalities shows the actual horror of the accidents. The model relates number of fatalities with the best set of explanatory factors as independent variables. With this objective in perspective, the research selects some possible factors as independent variables according to ARF (accident record form) of police that may contribute to road traffic accident fatalities. The independent variables in the modeling included factors such as- junction type, divider, roadway geometry, pedestrian location etc. To represent the non-linear relationship of fatalities with independent variables, Poisson regression model have been used. Therefore, delineation and subsequent improvement of these factors can improve the traffic safety at a given location.
\end{abstract}

Keywords: Fatalities, Pedestrian, Prediction model, Poisson regression model

\section{Introduction}

Road traffic accident, associated with rapid growth in motorization is a serious health problem amongst the poor and young people which causes heavy burden on the economy of a country. Among those who are killed on roads across the globe, about half of the fatalities constitute Vulnerable Road Users (VRUs), which include bicyclists, pedestrians, and motorcyclists [1]. In terms of pedestrian accidents, worldwide over 4,00,000 pedestrians die every year and over half of these deaths occur in low-income countries [2]. Due to their vulnerability, pedestrians are often called the "infantry of traffic". In urban areas of developing countries pedestrians are most at risk due to large amount of pedestrian and vehicle activity, especially in Dhaka, the capital of Bangladesh. Nearly $60 \%$ of urban trips involved walking in Dhaka and it is particularly prevalent for short trips predominantly because of economic reason and efficiency reason due to lack of sufficient transportation facilities [3]. Increasing rate of urban migration due to garment industries, better employment, all head offices, business opportunities, higher education opportunities, health facilities and higher income is constantly adding to the dramatic increase of pedestrian population in Dhaka metropolitan. With its large population Dhaka metropolitan in Bangladesh is facing a substantial increase in pedestrian fatality as effective strategies are not taken yet to ensure the safety of pedestrian. Even more distressing is the fact that children of this group are particularly at high risk. While many countries have strategies to mitigate pedestrian casualties, it is clear that many developing countries like Bangladesh are lacking in their safety amenities. So, to reduce the risk of pedestrians on roadway from fatalities is one of the main goals of transportation safety at hand for road safety improvement. Prediction of pedestrian fatalities associated with different factors can emphasize priority attention regarding pedestrian safety crisis. With this in mind, this study focuses on the development of pedestrian fatalities prediction model using different factors for Dhaka metropolitan in Bangladesh.

\section{Pedestrian safety crisis in Bangladesh}

Data used for analysis are collected from ARI (Accident Research Institute), BUET (Bangladesh University of Engineering and Technology) and analyzed using Microcomputer Accident Analysis Package Five (MAAP5) software. These data are collected by ARI as hard copies and soft copies from police as police is the primary data source for all reported accidents. ARI also edits the hard and soft copies to maintain MAAP5 database up to date. Data (1998 to 2008) reveal that almost 50 percent of all accidents involve pedestrians, of which nearly 80 percent are fatal accidents. Casualty analysis shows that, of the total road casualties 33.6 percent involved pedestrians, while 50 percent of all road traffic fatalities are pedestrian. Indeed, nearly 76 percent of pedestrian casualties are fatalities. Pedestrian casualty is also the highest on national highways (39.8\%) of which about 80 percent is fatality. According to location nearly 50 percent of pedestrian casualties occur on road side shoulders of which nearly 73 percent are fatalities while about 26 percent of pedestrian casualties occur on road center of which nearly 77 percent are fatalities. But percentage of pedestrian casualties according to their location changes from urban to rural area. According to junction type, about 75 percent of 
pedestrian casualties occur at not-junction or midblock sections. Among the vehicles bus (28.6\%) and heavy truck $(24.4 \%)$ are found to have high involvement in pedestrian casualties.

\section{Study area profile}

Dhaka is by far the largest urban area as well as the largest metropolitan area in Bangladesh. It is a metropolis of 2,161.17 square kilometers [4]. Population density in Dhaka is over 7,936.4 per square kilometer in the city area. Dhaka metropolitan city is one of the vulnerable cities of the world due to its high accident rates. Fig. 1 of casualty distribution among metropolitan areas in Bangladesh namely DMP (Dhaka Metropolitan), CMP (Chittagong Metropolitan), KMP (Khulna Metropolitan) and RMP (Rajshahi Metropolitan), proves this fact.

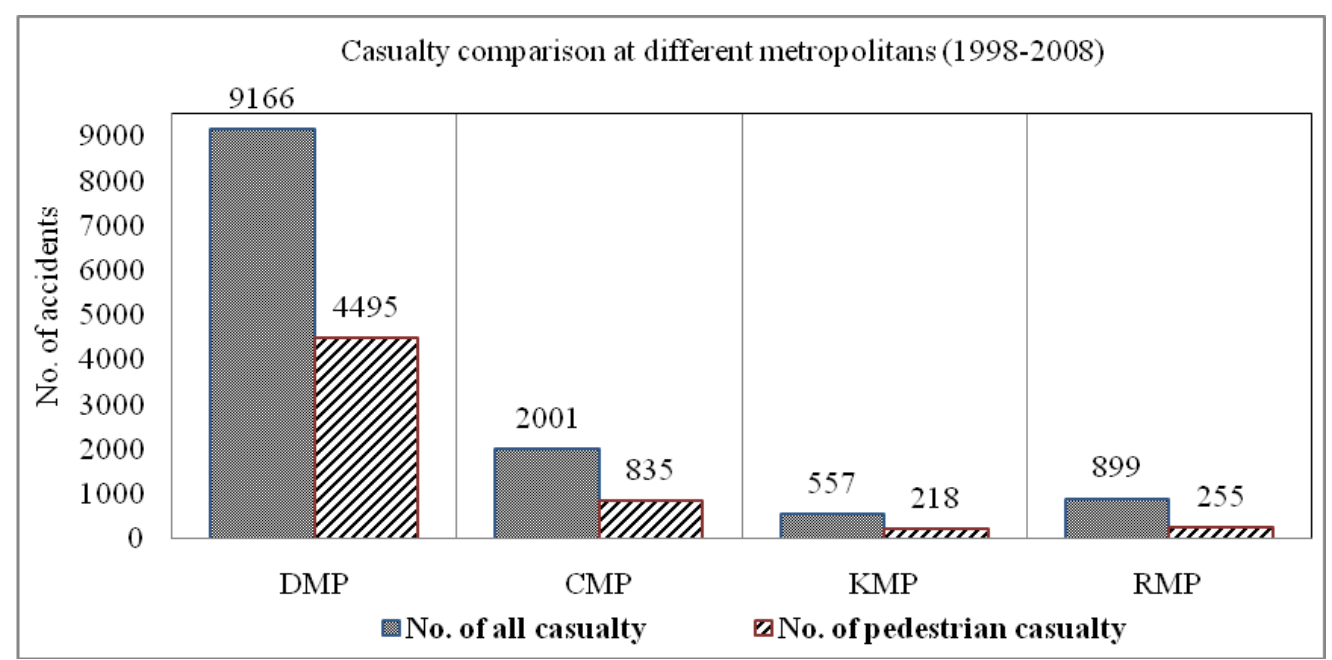

Figure 1: Casualty comparison at different metropolitans (1998-2008)

\section{Highlights of pedestrian casualty findings in DMP}

To know the pedestrian casualty scenario in DMP associated with different factors, number of pedestrian casualties according to junction type, traffic control, movement, divider, pedestrian action, pedestrian location and involved vehicle types are presented below.

- Pedestrian casualty according to junction type shows that at DMP, 68 percent casualty occurs at notjunction or mid-block sections. This is because pedestrian cross the road unconsciously according their will and also lacking of proper pedestrian facilities.

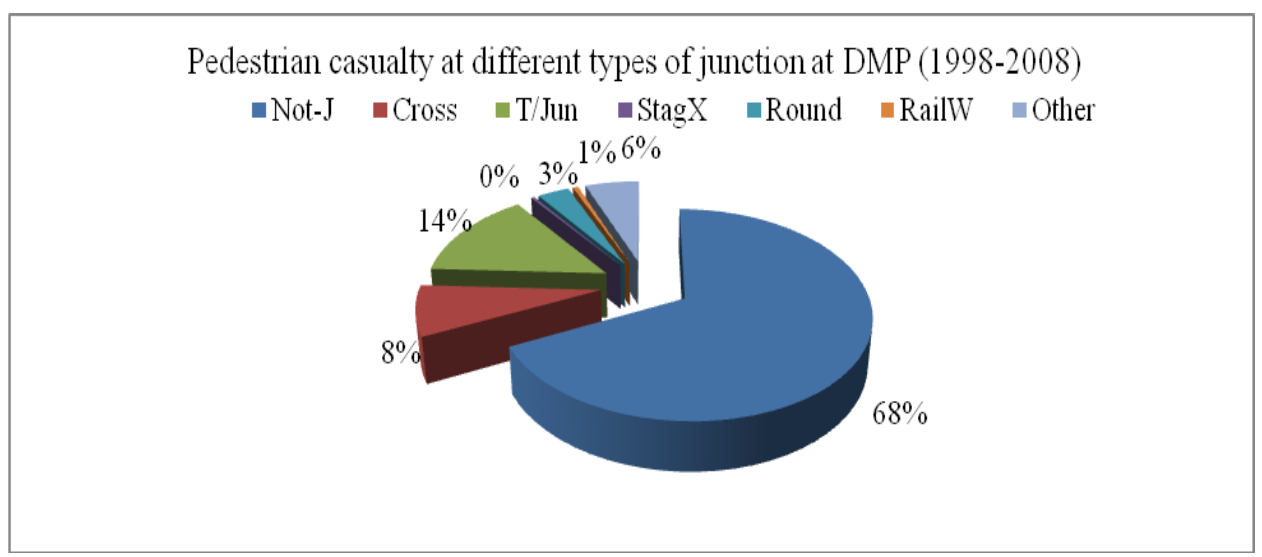

Figure 2: Pedestrian casualty at different types of junction at DMP

- Nearly $65 \%$ pedestrian casualty of DMP occurs when there is no traffic control.

- Nearly $79 \%$ pedestrian casualty of DMP occurs when 2-way traffic system prevails.

- Nearly 79\% pedestrian casualty of DMP occurs when there is divider on roadway.

- According to pedestrian action 52\% of pedestrian casualty of DMP occurs during road crossing.

- $54.5 \%$ pedestrian casualty of DMP occurs when pedestrians are on road center according to location.

- Highest percentage of pedestrian casualty occurs by bus (23.5\%) and heavy truck (22\%) at DMP. 


\section{Literature review}

It is almost impossible to predict the severity of a single event of an accident because of its rare and random nature. In reality, accident counts are sporadic, discrete and non negative. But researchers [5] have found that aggregation of a large number of accident data over a sufficiently wide area and/or long period of time tends to exhibit a level of predictability which can be described by means of statistical relationships. Poisson regression model represent a form of such relationships. Previous studies [6] have shown that multiple linear regression is not suitable for modelling such relationships, instead Poisson or Negative Binomial regression should be used. A previous study [7] used a Poisson model to relate exposure variables to crashes. Models were estimated using accident, travel mileage and environmental data from the Indiana Toll Road. A pooled model including all accidents revealed that accident occurrence increases with automobile vehicle miles of travel (VMT), truck VMT and hours of snowfall. A previous research [8] proposed a Poisson regression model to establish empirical relationships between truck accidents and key highway geometric design variables. Among the variables, final model suggested that annual average daily traffic per lane, horizontal curvature, and vertical grade were significantly correlated with truck accident involvement rate, where shoulder width had comparably less correlation. Most of these models were developed, however, for investigating the relationship of accident number with various factors. The literature review indicates a lack of studies investigating the relationship of fatalities with various possible factors.In this study, pedestrian injury severity was modeled as a function of various explanatory factors as independent variables using Poisson regression. Previously, no such research on the metropolitan cities has been conducted in Bangladesh context.

\section{Methodology}

For pointing a specific location of accident fatalities, two types of value is used, that is " $\mathrm{X}$ " co-ordinate and " $\mathrm{Y}$ " co-ordinate. In this model data preparation and analysis steps are given below.

- Parameters used in this model are Junction Type (JT), Roadway Geometry (RG), Divider (Div), Pedestrian Location (PL).

- "X" and "Y" co-ordinates are used for fixing location.

- In X and Y co-ordinate, per 1000 values is considered as a unit.

- Combining above parameters and co-ordinate, number of accident fatalities per year is determined for different combinations by using MATLAB coding.

- Then per year data from 1998 to 2008 is combined to get the fatalities for different parameter combinations.

- If any rows contain missing values then the entire row is deleted.

- Poisson regression is done by using SPSS.

- By trial and error (considering 95\% confidence interval) the best result is shown here for this model.

- Since, all variables in this model are of interest and expected to have some effect on accident fatalities; significance is kept as lower as possible.

\section{Model development}

During modeling several parameters were considered initially, but only parameters with good correlation were selected. Parameters used in this model are Junction Type (JT), Roadway Geometry (RG), Divider (Div) and Pedestrian Location (PL). Dummy variables which are assigned for each parameter types in the model are given below.

Table 1: Dummy variables for Junction Type (JT)

\begin{tabular}{|l|l|}
\hline Dummy variable assigned & Junction Type \\
\hline JT1 & Not Junction \\
\hline JT2 & Cross Junction \\
\hline JT3 & T- Junction \\
\hline JT4 & Staggered Junction \\
\hline JT5 & Roundabout Junction \\
\hline JT6 & Railway Junction \\
\hline JT7 & Other \\
\hline
\end{tabular}

Table 2: Dummy variables for Divider (Div)

\begin{tabular}{|l|l|}
\hline Dummy variable assigned & Divider \\
\hline Div1 & Yes \\
\hline Div2 & No \\
\hline
\end{tabular}

Table 3: Dummy variables for Roadway Geometry (RG)

\begin{tabular}{|l|l|}
\hline Dummy variable assigned & Roadway Geometry \\
\hline RG1 & Straight \\
\hline RG2 & Curve \\
\hline
\end{tabular}


A prediction model for pedestrian fatalities based on explanatory factors

\begin{tabular}{|l|l|}
\hline RG3 & Slope \\
\hline RG4 & Curve + Slope \\
\hline RG5 & Crest \\
\hline
\end{tabular}

Table 4: Dummy variables for Pedestrian Location (PL)

\begin{tabular}{|l|l|}
\hline Dummy variable assigned & Pedestrian Location \\
\hline PL1 & Pedestrian crossing \\
\hline PL2 & 50m- Within Pedestrian crossing \\
\hline PL3 & Divider \\
\hline PL4 & Center of road \\
\hline PL5 & Foot Path \\
\hline PL6 & Road Shoulder \\
\hline PL7 & Bus Stop \\
\hline
\end{tabular}

VIII. Model results and interpretation

From the SPSS analysis following Table 5, for Goodness of Fit was found.

Table 5: Goodness of Fit

\begin{tabular}{|l|l|}
\hline Goodness of Fit & Value/df \\
\hline Deviance & 0.461 \\
\hline Pearson Chi-Square & 0.651 \\
\hline
\end{tabular}

By considering the above Table 5, we see that deviance is 0.461 and Pearson chi-square is 0.651 . These values when close to 1, indicates a good goodness-of-fit. From the SPSS analysis following Table 6, for parameter estimates was found.

Table 6: Parameter estimates

\begin{tabular}{|l|l|l|}
\hline Parameter & Co-efficient & Hypothesis test (Significance) \\
\hline Intercept) & -0.779 & 0.463 \\
\hline$[\mathrm{JT}=1.0]$ & 0.353 & 0.001 \\
\hline$[\mathrm{JT}=2.0]$ & 0.084 & 0.512 \\
\hline$[\mathrm{JT}=3.0]$ & 0.100 & 0.388 \\
\hline$[\mathrm{JT}=4.0]$ & -0.127 & 0.715 \\
\hline$[\mathrm{JT}=5.0]$ & 0.096 & 0.547 \\
\hline$[\mathrm{JT}=6.0]$ & -0.143 & 0.640 \\
\hline$[\mathrm{JT}=7.0]$ & $0^{\mathrm{a}}$ & - \\
\hline$[\mathrm{Div}=1.0]$ & 0.250 & 0.000 \\
\hline$[\mathrm{Div}=2.0]$ & $0^{\mathrm{a}}$ & - \\
\hline$[\mathrm{RG}=1.0]$ & 0.234 & 0.816 \\
\hline$[\mathrm{RG}=2.0]$ & 0.000 & 1.000 \\
\hline$[\mathrm{RG}=3.0]$ & -0.353 & 0.804 \\
\hline$[\mathrm{RG}=4.0]$ & -0.353 & 0.774 \\
\hline$[\mathrm{RG}=5.0]$ & $0^{\mathrm{a}}$ & - \\
\hline$[\mathrm{PL}=1.0]$ & 0.188 & 0.622 \\
\hline$[\mathrm{PL}=2.0]$ & 0.204 & 0.723 \\
\hline$[\mathrm{PL}=3.0]$ & 0.335 & 0.585 \\
\hline$[\mathrm{PL}=4.0]$ & 0.529 & 0.135 \\
\hline$[\mathrm{PL}=5.0]$ & 0.119 & 0.786 \\
\hline$[\mathrm{PL}=6.0]$ & 0.345 & 0.332 \\
\hline$[\mathrm{PL}=7.0]$ & $0^{\mathrm{a}}$ & - \\
\hline$(\mathrm{Scale})$ & $1^{\mathrm{b}}$ & \\
\hline & & \\
\hline
\end{tabular}

The higher the positive value of a co-efficient, the higher it is responsible for causing accident fatalities. From the above table we see that the co-efficient value of JT1, PL3, PL4 and Div2 is higher than the other. So, we can conclude that these are the most vulnerable factors for casing pedestrian fatalities. From the above analysis, the final equation obtained for pedestrian fatalities:

Pedestrian fatalities (per year) $=\mathrm{e}^{\mu}$

Where,

$\mu=-0.779+0.353 \mathrm{JT} 1+0.084 \mathrm{JT} 2+0.1 \mathrm{JT} 3-0.127 \mathrm{JT} 4+0.096 \mathrm{JT} 5-0.143 \mathrm{JT} 6+0.250 \mathrm{DIV} 1+0.234 \mathrm{RG} 1-0.353 \mathrm{RG} 3-$ 0.353RG4+0.188PL1+0.204PL2+0.335PL3+0.529PL4+0.119PL5+0.345PL6

\section{Conclusion}

Transportation planners especially transportation safety provider may use this model to evaluate the vulnerability of pedestrian associated with different factors in Dhaka metropolitan city. Also this model may be useful at the stage of planning potential countermeasures. 


\section{Reference}

[1]. WHO, Global status report on road safety: Time for action, 2009.

[2]. H.M. Ahsan, M.M. Khan and M.A. Raihan, Pedestrian the Most Vulnerable Group in Road Traffic Accidents, CD proceedings of $1^{\text {st }}$ International Conference on Advances in Civil Engineering (ICACE), 12 -14 December 2012, CUET, Chittagong, Bangladesh.

[3]. R. Rahman, S.M.S. Mahmud, M.M. Hoque and S.N. Ahmed, Pedestrian accident characteristics in Dhaka : A comparative investigation. Proceedings of International Conference on Road Safety in Developing Countries, ARC, BUET, 22-24 August 2006, 84-90.

[4]. Bangladesh Bureau of Statistics, BBS report, 2011.

[5]. L. Fridstrom, J. Ifver, S. Ingebrigsten, R. Kulmala and L.K. Thomsen, Measuring the contribution of randomness, exposure, weather and daylight to the variation in road accident counts, Accident Analysis and Prevention, 27(1), 1995, 1-20.

[6]. J. Milton and F. Mannering(1998), The relationship among highway geometrics, traffic related elements and motor-vehicle accident frequencies. Transportation, 25, 1998, 395-413.

[7]. P.P Jovanis, H.L Chang, Modelling the relationship of accidents to miles travelled, Transportation Research Record, Washington, DC., No.1068, 1986, 42-51.

[8]. S.P. Miaou, P.S. Hu, T. Wright, A.K. Rathi and S. Davis, Relationships between truck accidents and highway geometric design: a Poisson regression approach, Transportation Research Record No. 1376, 1992, 10-18. 